\title{
GENERALIZED METHOD FOR FORMING PLANE ISOTROPIC CURVES
}

\section{A. V. Nesvidomin, Candidate of Technical Sciences, Senior Lecturer}

\author{
National University of Life and Environmental Sciences of Ukraine
}

\section{E-mail: a.nesvidomin@gmail.com}

\begin{abstract}
The process of modeling the temperature distribution on surfaces, applying an image to curved areas with minimal distortion requires the formation of isometric grids on the plane and on the surface. One of the common ways to form planar isometric networks is to use the functions of a complex variable and planar isotropic curves, followed by separation of the real and imaginary parts. The development of computer models for the interactive search and analysis of isometric networks according to various initial geometric conditions provides a generalized method for their formation with the possibility of varying their shape and position.

It is proposed to use an isotropic vector for the formation of flat isotropic curves, which ensured a single sequence of analytical calculations according to the following initial conditions: 1) selection of an arbitrary function of a real argument; 2) a given parametric equation of a plane curve; 3) a given polar equation of a plane curve.

Since the analytical calculations of the derivation of the parametric equation of a plane isotropic curve and the corresponding isometric grid are rather laborious, their execution is carried out in the environment of the Maple symbolic algebra. To this end, the corresponding software has been created, which interactively allows you to select the function of a real argument, a parametric or polar equation of a plane guide curve. All subsequent stages of analytical transformations to form an isotropic curve and the corresponding isometric grid are carried out automatically. An interactive model for the formation and analysis of plane isotropic curves with various initial conditions has been created, which has shown its effectiveness, which is confirmed by the given examples of plane isometric grids for specific functions of the real parameter, plane curves in the parametric and polar form of their job.
\end{abstract}

Key words: isotropic vector, plane isotropic curve, plane isometric grid, real and complex planes, equation of curves in the complex plane

Topicality. The existence of different ways of forming flat isotropic curves complicates their computer implementation under predetermined initial conditions. The solution to this problem is the development of a generalized method for forming isotropic curves by an arbitrary analytical function, a guide flat curve in the parametric and polar forms. 
Analysis of recent research and publications. The construction of flat isotropic curves according to the initial parametric equations of the flat curve is given in [2]. The formation of isometric grids and their application for drawing images with minimal distortion is shown in [3].

Formulation of the goals of the article. To reveal a generalized method of forming flat isotropic curves under different initial conditions, to give examples of their application in the construction of flat isometric grids in the Maple software[1].

Materials and methods of research. The complex number $z=1+I$ on the complex plane is represented by a point with coordinates $[1, I]$, where $I=\sqrt{-1}-$ is an imaginary unit. Suppose we have a vector a with coordinates:

$$
\boldsymbol{a}=[1, \pm I] \text {. }
$$

Since the length of the vector $a$ on the real plane is zero $\sqrt{1^{2}+( \pm I)^{2}}=0$, such a vector is isotropic.

Results of the studies and their discussion. Formation of isotropic curves using the analytical function $f(t)$ of a valid argument $t$.

Suppose we have any function $f(t)$ of a valid argument $t$. Its multiplication by the isotropic vector $\boldsymbol{a}$ leads to the isotropic line $\boldsymbol{r}(t)$ along the vector $\boldsymbol{a}$ in the following parametric form:

$$
\boldsymbol{r}(t)=f(t) \boldsymbol{a}=f(t)[1, \pm I]
$$

Since it is not possible to visualize an isotropic curve (2) that has zero length on a real plane, its construction can be carried out either through its real or imaginary part, or through the construction of isometric grids that these curves generate.

The sequence of formation of flat isometric grids using an isotropic curve (2) is as follows:

in the isotropic curve $r(t)$ replace the real argument $t$ with a given function $f(z)$ of the complex variable $z=u+I v$ obtain the curve $r(f(u+I v))$ on the complex plane:

$$
\boldsymbol{r}(f(u+I v))=\boldsymbol{r}\left[f_{t}(f(u+I v)), \pm I \cdot f_{t}(f(u+I v))\right]
$$


separate only the real parts of the curve $r(f(u+I v))$ :

$$
\boldsymbol{R}_{R}(u, v)=\boldsymbol{R}[\operatorname{Re}(r(f(z)))]
$$

or separate only the imaginary parts of the curve $r(f(z u+I v))$ :

$$
\boldsymbol{R}_{I}(u, v)=\boldsymbol{R}[\operatorname{Im}(\boldsymbol{r}(f(u+I v)))] .
$$

The flat isometric grids (4) and (5) are congruent and rotated at an angle of $90^{\circ}$. Figure 1 constructs isometric grids (4) for the functions $f(t)=\sin (t), \sinh (t), \operatorname{sech}(t)$.

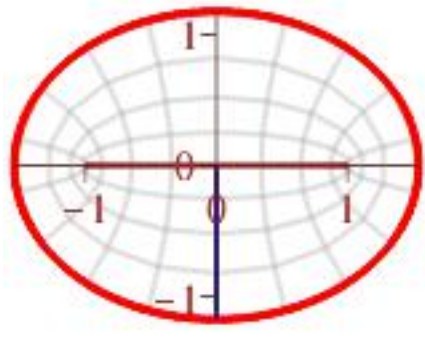

$a$

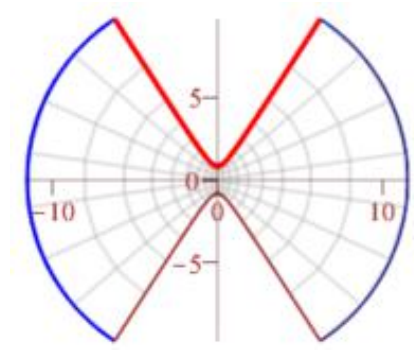

$b$

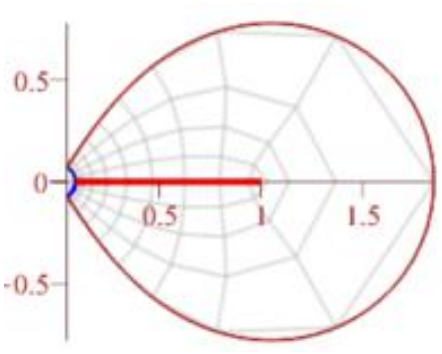

$c$

Fig.1. Flat isometric grids for functions $\boldsymbol{f}(\boldsymbol{t})$ :

$$
a-\sin (t) ; b-\sinh (t) ; c-\operatorname{sech}(t)
$$

Formation of isotropic curves using parametrically given flat curves $\boldsymbol{\mu}(t)=[x(t), y(t)]$ дійсної змінної $t=t_{1 \times} t_{2}$.

Since each point $[\mathrm{x}, \mathrm{y}]$ of the plane represents the complex number $z=x+I y$, then the parametric equation of the plane curve $\boldsymbol{\mu}(t)$ on the complex plane will be expressed by the following expression:

$$
f(t)=x(t)+I y(t), t=t_{1} . . t_{2} .
$$

Multiplying the expression $\mathrm{f}(\mathrm{t})$ of the real argument $t$ by the isotropic vector $\boldsymbol{a}$ leads to the isotropic line $\boldsymbol{r}(t)$ in parametric form:

$$
\boldsymbol{r}(t)=f(t) \cdot \boldsymbol{a}=[x(t)+I y(t), I x(t)-y(t)] .
$$

Isometric grids (4) for flat curves $\boldsymbol{\mu}(t)=\left[t, t^{2}\right],[t, \sin (t)],[\sin (t), \sinh (t)]$ are constructed in Fig.2.

Formation of isotropic curves using plane curves $\rho(t)$ given in the polar coordinate system.

The equation of any curve $\rho(t)$ in the polar coordinate system on the complex plane will have the following trigonometric shape: 


$$
f(t)=\rho(t)(\cos (t)+I \sin (t)) \text {. }
$$

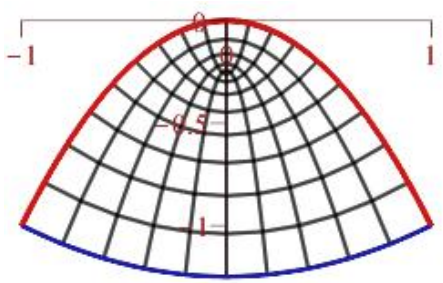

$a$

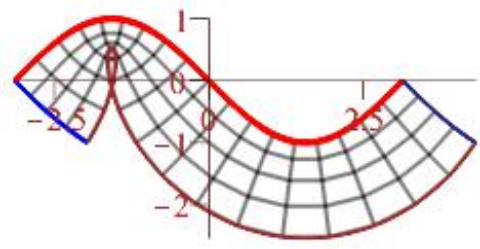

$b$

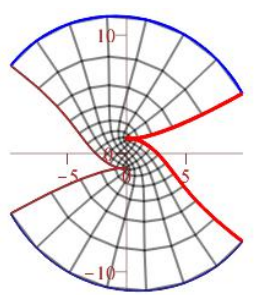

$c$

Fig.2. Isometric grids along the guide flat curves $\boldsymbol{\mu}(\boldsymbol{t})$ : $a-\left[t, t^{2}\right] ; b-[t, \sin (t)] ; c-[\sin (t), \sinh (t)$

Multiplying the expression $f(t)$ of curve (8) by the isotropic vector $\boldsymbol{a}$ leads to the isotropic liner $(t)$ in parametric form:

$$
\boldsymbol{r}(t)=\rho(t)[\cos (t)+I \cdot \sin (t),-I \cdot(\cos (t)+I \cdot \sin (t))] .
$$

Figure 3 constructs flat isometric grids by equation (9) for the characteristic curves $\rho(t)$ given in the polar coordinate system.
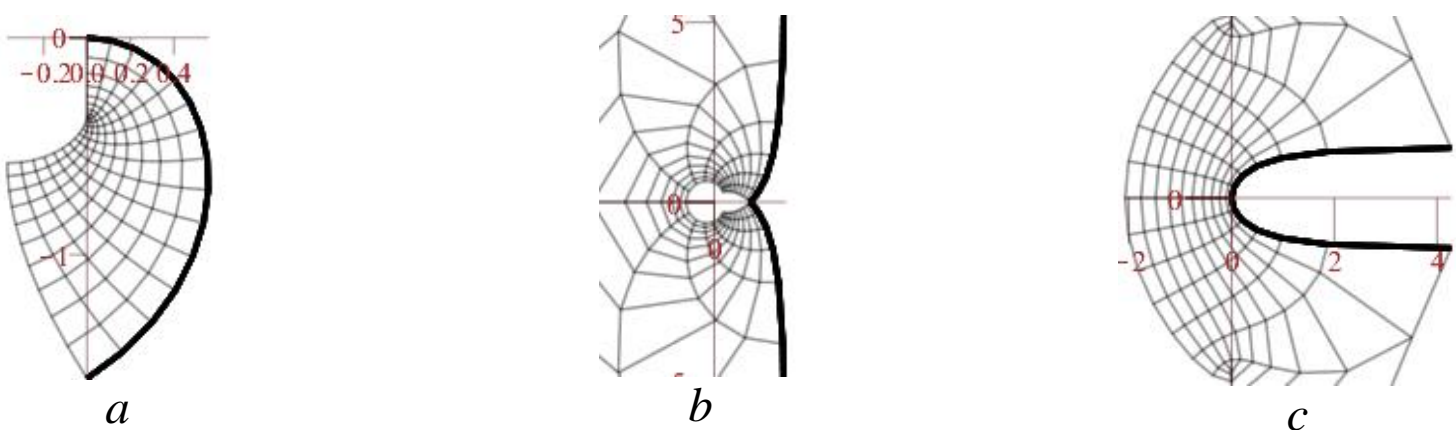

Fig.3. Flat isometric grids along guide curves in the polar coordinate system under the condition $a=1$ :

$a-$ Archimedes spiral $\rho(t)=a t ; b-$ strophoid $\rho(t)=a \frac{1+\sin (t)}{\cos (t)}$;

$$
c-\operatorname{kappa} \rho(t)=\operatorname{actg}(t)
$$

The curve $\rho(t)$ in the polar coordinate system on the complex plane can also be represented in the exponential form $f(t)=\rho(t) e^{I t}$. Multiplying the expression $f(t)$ by the isotropic vector a leads to the isotropic line $\boldsymbol{r}(t)$ in parametric form:

$$
r(t)=\rho(t)\left[e^{I t}, I e^{I t}\right] .
$$

The isotropic curve (9) forms flat isometric grids congruent to the isotropic curve (8). 
All the above methods fit into a single scheme (Table 1), in accordance with which a computer model of the formation of isometric grids under different initial conditions was created.

1. Single scheme

\begin{tabular}{|c|c|c|c|}
\hline Option & $\begin{array}{l}\text { Reassignment } f(t) \text { in } \\
\text { complex form }\end{array}$ & $\begin{array}{l}\text { Isotropic } \\
\text { curve } \boldsymbol{r}(t)\end{array}$ & $\begin{array}{c}\text { Isometric } \\
\text { grid }\end{array}$ \\
\hline function $f(t)$ & $f(t)$ & {$[f(t), I \cdot f(t)]$} & \multirow{3}{*}{$\boldsymbol{R}_{R}(u, v)$} \\
\hline $\begin{array}{c}\text { curve } \\
{[x(t), y(t)]}\end{array}$ & $x(t)+I \cdot y(t)$ & {$\left[\begin{array}{l}x(t)+I y(t), \\
I \cdot x(t)-y(t)\end{array}\right]$} & \\
\hline curve $\rho(t)$ & $\rho(t) \cdot e^{I t}$ & $\rho(t)\left[e^{I t}, I \cdot e^{I t}\right]$ & \\
\hline
\end{tabular}

Conclusions and Perspective The use of the isotropic vector $[1, \pm I]$ allowed to reduce the analytical description of the formation of a flat isotropic curve and the corresponding isometric grid to 4 main operations: 1) the choice of the guide curve; 2) reassignment of this curve in a complex form; 3 ) multiplication of the complex equation of the curve by an isotropic vector; 4) separation of real or imaginary parts of an isotropic curve.

\section{Список використаних джерел}

1. Аладьев В.З., Бойко В.К., Ровба Е.А. Программирование и разработка приложений в Мaple. Гродно-Таллин, 2007. 458 с.

2. Несвідоміна О.В. Побудова плоских ізометричних сіток за наперед заданими плоскими кривими. Вісник Херсонського національного технічного університету. Херсон: ХНТУ, 2017. Вип.3(620). Т.2. С. 196-199.

3. Пилипака С.Ф., Кремець Т.С., Несвідоміна О.В. Конформне відображення растрових написів на плоскі криволінійні області. Сучасні проблеми моделювання: збірник наук. праць МДПУ ім. Б. Хмельницького. Мелітополь: МДПУ, 2018. Вип. 13. C. $124-130$.

\section{References}

1. Alad'yev, V. Z., Boyko, V. K., Rovba, E. A. (2007). Programmirovaniye i razrabotka prilozheniy $\mathrm{v}$ Maple [Programming and application development in Maple]. Grodno-Tallin, 458.

2. Nesvidomina, O. V. (2017). Pobudova ploskykh izometrychnykh sitok za napered zadanymy ploskymy kryvymy [Construction of flat isometric grids according to predefined flat curves]. Visnyk Khersonskoho natsionalnoho tekhnichnoho universytetu. Kherson: KhNTU, 3(620), Vol. 2, 196-199. 
3. Pylypaka, S. F., Kremets, T. S., Nesvidomina, O. V. (2018). Konformne vidobrazhennia rastrovykh napysiv na ploski kryvoliniini oblasti [Conformal mapping of raster inscriptions on flat curved areas]. Suchasni problemy modeliuvannia: zbirnyk nauk. prats MDPU im. B. Khmelnytskoho. Melitopol: MDPU, 13, $124-130$.

\section{УЗАГАЛЬНЕНИЙ СПОСІБ ФОРМУВАННЯ ПЛОСКИХ ІЗОТРОПНИХ КРИВИХ}

\section{А. В. Несвідомін}

Анотація. Процес моделювання розподілу температури на поверхнях, нанесення зображення на криволінійні області з мінімальними спотвореннями потребує формування ізометричних сіток на площині та на поверхні. Одним із потирених способів формування плоских ізометричних сіток $є$ використання функиій комплексної змінної та плоских ізотропних кривих з подальшим відокремленням дійсної та уявної частин. Розробка комп'ютерних моделей інтерактивного пошуку та аналізу ізометричних сіток за різними вихідними геометричними умовами передбачає узагальнений спосіб їх формування 3 можливістю варіювання їх формою та положенням.

Запропоновано використати ізотропний вектор для формування плоских ізотропних кривих, щзо забезпечило єдину послідовність аналітичних викладок за такими вихідними умовами: 1) вибору довільної функиії дійсного аргументу; 2) заданого параметричного рівняння плоскої кривої; 3) заданого полярного рівняння плоскої кривої.

Оскільки аналітичні викладки виведення параметричного рівняння плоскої ізотропної кривої та відповідної ізометричної сітки є досить трудомісткими, то їх виконання здійснюється в середовищі символьної алгебри Maple. 3 иією метою створено відповідне програмне забезпечення, яке в інтерактивному режимі дозволяє здійснити вибір функиї дійсного аргументу, параметричне чи полярне рівняння плоскої напрямної кривої. Всі наступні етапи аналітичних перетворень з формування ізотропної кривої та відповідної ізометричної сітки здійснюється автоматично. Створена інтерактивна модель формування та аналізу плоских ізотропних кривих за різними вихідними умовами показала ї̈ ефективність, що підтверджено наведеними прикладами плоских ізометричних сіток для конкретних функиій дійсного параметра, плоских кривих в параметричній та полярній формі їх задання.

Ключові слова: ізотропний вектор, плоска ізотропна крива, плоска ізометрична сітка, дійсна та комплексна площина, рівняння кривих в комплексній площині

\section{ОБОБЩЕННЫЙ СПОСОБ ФОРМИРОВАНИЯ ПЛОСКИХ ИЗОТРОПНЫХ КРИВЫХ}

\section{А. В. Несвидомин}

Аннотация. Прочесс моделирования распределения температуры на поверхностях, нанесение изображения на криволинейные области с минимальными искажениями требует формирования изометрических сеток на плоскости и на 
поверхности. Одним из распространенных способов формирования плоских изометрических сетей является использование функиий комплексной переменной и плоских изотропных кривых с последующим отделением действительной и мнимой частей. Разработка компьютерных моделей интерактивного поиска и анализа изометрических сетей по различным исходным геометрическими условиями предусматривает обобщенный способ их формирования с возможностью варьирования их формой и положением.

Предложено использовать изотропный вектор для формирования плоских изотропных кривых, что обеспечило единую последовательность аналитических выкладок по таким исходным условиям: 1) выбора произвольной функции действительного аргумента; 2) заданного параметрического уравнения плоской кривой; 3) заданного полярного уравнения плоской кривой.

Поскольку аналитические выкладки вывода параметрического уравнения плоской изотропной кривой и соответствующей изометрической сетки являются достаточно трудоемкими, то их выполнение осуществляется в среде символьной алгебры Maple. С этой целью создано соответствующее программное обеспечение, которое в интерактивном режиме позволяет осуществить выбор функции действительного аргумента, параметрическое или полярное уравнение плоской направляющей кривой. Все последующие этапы аналитических преобразований по формированию изотропной кривой и соответствующей изометрической сетки осуществляется автоматически. Создана интерактивная модель формирования и анализа плоских изотропных кривых различными исходными условиями показала ее эффективность, что подтверждено приведенными примерами плоских изометрических сеток для конкретных функиий действительного параметра, плоских кривых в параметрической и полярной форме их задания.

Ключевые слова: изотропный вектор, плоская изотропная кривая, плоская изометрическая сетка, действительная и комплексная плоскости, уравнение кривых в комплексной плоскости 